$\underline{\text { P-2 }}$

\title{
Evaluation the Anti-Inflammatory Activity of Some Eastern Libyan Medicinal Plants
}

\author{
Abdulmottaleb E Zetrini ${ }^{1, *}$, Hamza M flafel ${ }^{1}$, Sami J Alsabri ${ }^{1}$, Khalid Milad Aburas ${ }^{1}$, Akram E \\ Mohamed $^{1}$, Nouri B. Rmeli ${ }^{2}$ and Abdul Gbaj ${ }^{1,3}$ \\ ${ }^{l}$ National Medical Research Centre, Zawia, Libya; ${ }^{2}$ Tripoli University, Faculty of pharmacy, Department of Natural \\ Products, Tripoli, Libya; ${ }^{3}$ Tripoli university, faculty of pharmacy department of medicinal chemistry, Tripoli Libya; \\ E-mail:ph_abdo1986@yahoo.com
}

Four Libyan medicinal plants Olea Europaea (Oleacea), Cistus Incanus (Cistaceae), Launaea Residifolia (Asteraceae), Cistus Parviflorus ,Lam(Cistaceae) were collected from EL - jabal EL-akhdar eastern Libya in April 2010 .after identified the plants were extracted using microwave technique by three solvents (methanol-ethyl acetate -hexane ) .these plants were studied for anti-inflammatory activity using carrageenan induced mice odema model. The plant extracts of different solvents were administered at dose $250 \mathrm{mg} / \mathrm{kg}$ of the body weight intraperitoneally. among these extracts, Cistus Parviflorus ,Lam extract especially methanolic and ethyl acetate extracts gave the most significant \% inhibition of inflammation after $3 \mathrm{hr}(57.4 \pm 1.9$ $65.5 \pm 2.5$ respectively) this effect was better than standard reference aspirin (55.1 \pm 1.9$)$

Keywords: Anti-inflammatory, Olea Europaea, Cistus Incanus, Launaea Residifolia, Cistus Parviflorus, Lam. 